\title{
Desigualdad y apertura comercial: un análisis econométrico para la economía ecuatoriana
}

\section{Inequality and trade openness: an econometric analysis for Ecuador}

\author{
Diana Morán Chiquito* \\ dianagar16@hotmail.com
}

\section{Resumen}

El presente artículo tiene como objetivo medir el impacto de la apertura comercial sobre la desigualdad en la distribución del ingreso en la economía ecuatoriana a partir de la década de los noventa. A través de un modelo VAR se estudia la relación existente entre la liberalización comercial y la desigualdad. Los hallazgos sugieren que la apertura comercial determina la evolución de la desigualdad de la distribución de los ingresos. A un aumento en el flujo de comercio externo corresponde un aumento en la desigualdad distributiva.

\section{Palabras clave}

Comercio Exterior, desigualdad, distribución del ingreso, Ecuador.

\section{Abstract}

This paper aims to measure the impact of reforms on trade liberalization on income inequality in the ecuadorian economy since the nineties. Through a VAR model the relationship between economic growth, trade liberalization and inequality is studied. The findings suggest that trade liberalization determines the inequality. An increase in the foreign trade generates an increase in income inequality.

\section{Keywords}

Foreign trade, inequality, income distribution, Ecuador.

Forma sugerida de citar: Morán Chiquito, Diana M. (2015). Apertura comercial y desigualdad. Un análisis econométrico para la economía ecuatoriana. Revista Retos, 10(2), pp.163-175.

\footnotetext{
* Maestra en Ciencias Económicas. Profesora-Investigadora de la Facultad de Ciencias Económicas, Universidad de Guayaquil.
} 


\section{Introducción}

Al finalizar la década de los ochenta, los países latinoamericanos experimentaron procesos con profundas reformas económicas enfocadas en la liberalización comercial y de capitales, así como en el mantenimiento equilibrado de la balanza de pagos. Si bien la apertura económica ha resultado ser una fuente de crecimiento del producto, también ha aumentado la volatilidad de las economías latinoamericanas frente a choques externos. Así, en este orden de ideas es natural plantearse la siguiente interrogante: ¿Pueden ser estas reformas la razón o pueden estas constituirse en un factor explicativo del aumento de la desigualdad en la distribución del ingreso?

En la literatura que aborda esta problemática se pueden encontrar estudios que argumentan que la liberalización comercial favorece el crecimiento económico y promueve una distribución del ingreso más igualitaria. De acuerdo con este enfoque, se aplicaron una serie de políticas orientadas a la liberalización del comercio exterior, la apertura al capital externo, y la simplificación de todas las formas de intercambio externo, a excepción de la movilidad de la mano de obra ${ }^{1}$.

En contraposición a este enfoque, existen autores que señalan que

1 Posteriormente estos instrumentos y otros que fueron adicionados se condensaron en lo que se denominó el Consenso de Washington (1989). la apertura comercial, sustentada en la teoría del comercio internacional de las ventajas comparativas, ha repercutido negativamente en la desigualdad de ingresos en los países en vías de desarrollo.

El trabajo se estructura de la siguiente manera. En la primera parte se realiza un análisis sobre la economía ecuatoriana durante el período de estudio. En la segunda sección se describe la metodología utilizada. En el tercer apartado se muestran los resultados de las estimaciones. Finalmente se aborda la discusión de los hallazgos y se presentan las conclusiones.

\section{Situación de la economía ecuatoriana, 1995-2011}

El Ecuador durante la segunda mitad de la década de los noventa atravesó por conflictos sociales y políticos, y desastres naturales que derivaron en la peor crisis económica del país durante su etapa republicana. En lo político, entre 1996 y 2005 el cargo de Jefe de Estado fue ocupado por siete mandatarios; la inestabilidad política que marcó esta etapa, caracterizada por una crisis de gobernanza, repercutió negativamente en la economía observándose a través de la fuga de capitales financieros y la reducción de la inversión extranjera directa. En lo social, la guerra fronteriza contra Perú en 1995, movilizó distintos sectores de la sociedad, el descontento social que manifestaba la población se centraba en la fuga de recursos que se canalizaban al mantenimiento del conflicto 
bélico en detrimento del gasto público social y el abandono a los sectores más necesitados.

Además, el fenómeno climatológico del Niño que afectó a la geografía ecuatoriana durante 1997 se tradujo en una desmejora en el bienestar de la población más pobre, en especial de los campesinos rurales que perdieron sus cultivos. Por su parte la población asentada en las grandes ciudades se vio afectada por las inundaciones, la escasez de alimentos, y el daño sufrido en sus viviendas. A todo lo anterior se sumó la crisis financiera que estalló a finales del siglo $\mathrm{XX}$; crisis que tuvo como detonante el congelamiento de depósitos (o feriado bancario) y que culminó en la dolarización de la economía.

\section{Metodología}

Para medir el efecto de la liberalización comercial sobre la desigualdad de ingresos, se utilizará como herramienta metodológica los modelos de autorregresión vectorial ${ }^{2}$ desarrollados por el Premio Nobel Christopher Sims. A diferencia de los modelos de ecuaciones simultáneas, los modelos VAR parten del supuesto de interdependencia y exogeneidad entre las variables del modelo (Sims, 1980). Los modelos tradicionales de la teoría económica requieren que

2 También denominados modelos VAR. previamente se establezca una diferenciación entre dos categorías de variables; de acuerdo con Sims, si hay verdadera simultaneidad entre un conjunto de variables, todas deben tratarse en igualdad de condiciones, de modo que no exista ninguna distinción a priori entre variables endógenas y exógenas (Gujarati, \& Porter, 2010). Sobre este punto, surgen dificultades para decidir que variables serán tomados como explicadas y cuáles como explicativas, dificultad que se soluciona con la implementación de los vectores autorregresivos (Maddala, 1996).

Los modelos VAR permiten analizar el impacto de un cambio no previsto en una de las variables sobre las demás variables del modelo. Con este fin se utilizan herramientas como las funciones de impulso respuesta y las descomposiciones de varianza.

Matemáticamente los modelos VAR se expresan como sigue:

$$
\begin{aligned}
& Y_{t}=C+A_{0} Y_{t}+A_{1} Y_{t-1}+ \\
& +A_{\rho} Y_{t-\rho}+B X_{t}+\varepsilon_{t}
\end{aligned}
$$

Donde $Y_{t}$, es un vector de variables endógenas de dimensión $n x 1 ; C$, es un vector de términos constantes de dimensión $n \times 1 ; A_{i}$, es una matriz de coeficientes de dimensión nxn; $\rho$, número de rezagos; $X_{t}$, es un vector de variables dummy; $B$, es una matriz 
de coeficientes de dimensión $n x 3 ; \varepsilon_{t}$, es el vector de errores de dimensión $n x 1$; exentos de correlación serial y heteroscedasticidad.
De (1), se obtiene la forma reducida del modelo de autorregresión vectorial:

$$
\begin{aligned}
& Y_{t}-A_{0} Y_{t}=C+A_{1} Y_{t-1}+\ldots \ldots \ldots \ldots+A_{\rho} Y_{t-\rho}+B X_{t}+\varepsilon_{t} \\
& \left(I_{n}-A_{0}\right) Y_{t}=C+A_{1} Y_{t-1}+\ldots \ldots \ldots \ldots+A_{\rho} Y_{t-\rho}+B X_{t}+\varepsilon_{t} \\
& \left(I_{n}-A_{0}\right)\left(I_{n}-A_{0}\right)^{-1} Y_{t}=\left(I_{n}-A_{0}\right)^{-1}\left[C+A_{1} Y_{t-1}+\ldots \ldots \ldots \ldots+A_{\rho} Y_{t-\rho}+B X_{t}+\varepsilon\right. \\
& Y_{t}=C_{0}+\Gamma_{1} Y_{t-1}+\Gamma_{2} Y_{t-2} \ldots \ldots \ldots \ldots .+\Gamma_{\rho} Y_{t-\rho}+\Psi X_{t}+\eta_{t}
\end{aligned}
$$

La ecuación (2), describe un modelo VAR en su forma reducida. donde:

$$
\begin{aligned}
& C_{0}=\left(I_{n}-A_{0}\right)^{-1} C \\
& \Gamma_{i}=\left(I_{n}-A_{0}\right)^{-1} A_{i} \\
& \Psi=\left(I_{n}-A_{0}\right)^{-1} B \\
& \eta_{t}=\left(I_{n}-A_{0}\right)^{-1} \varepsilon_{t} \\
& i=1,2,3, \ldots \ldots, \rho
\end{aligned}
$$

Las series de tiempo utilizadas han sido obtenidas de dos fuentes. Los datos correspondientes al Índice de Gini provienen de la Standardized World Income Inequality Database 3.0 (SWIID), la serie cubre el periodo de 1990 al 2011 en datos anuales. La información correspondiente al PIBpc es tomada de la base estadística de la CEPAL; las series correspondientes a las exportaciones, las importaciones y el PIB real, de las cuales se deriva el coeficiente de apertura externa, se toman de la base estadística de la CEPAL.

A continuación se realiza una breve explicación de cada una:

1. Producto Interno Bruto por habitante (PIBPC), logaritmo del PIB por habitante expresado en términos constantes.

2. Coeficiente de Gini (Gini), coeficiente utilizado para medir la desigualdad de ingresos, el cual es un número entre 0 y 1 , en donde el 0 corresponde a perfecta igualdad, esto es, todas las personas tienen el mismo ingreso; y el 1 se corresponde a la perfecta desigualdad, una sola persona concentra todo el ingreso.

3. Apertura Comercial (t_ratio), se define como las exportaciones más las importaciones divididas para el PIB real, utilizada como una variable proxy de medida de apertura comercial. 


\section{Gráfico 1: Evolución del Coeficiciente de Gini, Producto Interno Bruto per capita y Apertura comercial. 1990-2011}

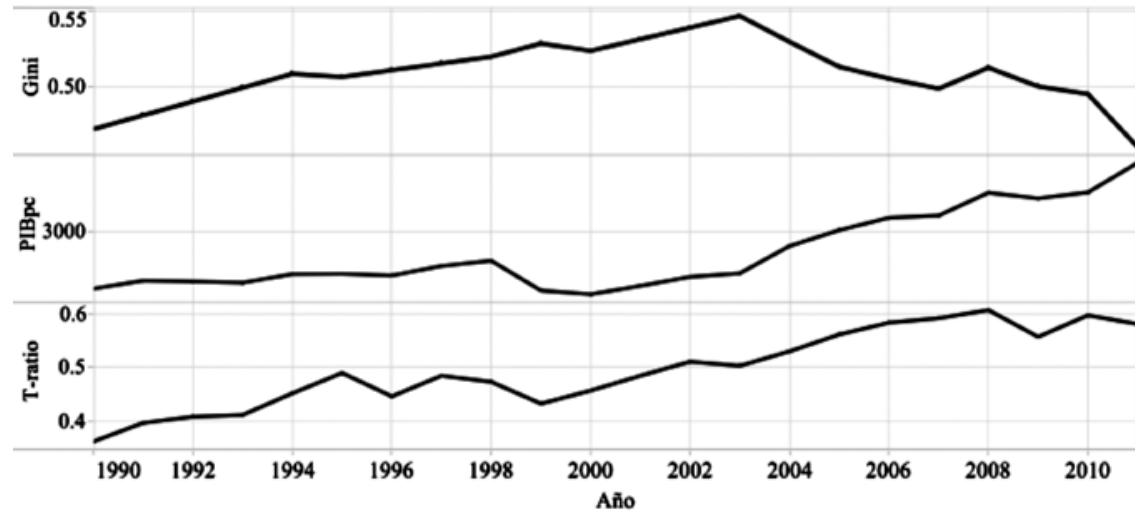

En el gráfico 1 se presenta la evolución de las tres variables analizadas. El PIBpc muestra una tendencia relativamente estable. Sin embargo, y como se ha mencionado en líneas anteriores, a finales de esta década el país enfrentó shocks negativos que desembocaron en la peor crisis de su historia (Morán, 2014). En este contexto de desestabilización macroeconómica, se observa una caída en el nivel del PIBpc, situación que se mantiene hasta el 2003. Es a partir del año 2004 que se observa un repunte en el crecimiento del PIBpc, la favorable coyuntura externa que propició el aumento del precio del petróleo lideró la dinámica de crecimiento

Por su parte la tendencia mostrada por la variable asociada a la apertura comercial es de orden ascendente durante todo el período que abarca el estudio. Se debe precisar que tal incremento en la apertura de la economía se debe en sumo grado al crecimiento de las exportaciones vinculada a los elevados precios del petróleo en el mercado mundial, principalmente a partir del 2000 (Ordóñez, 2006). El Ecuador, como lo señalan Acosta (2005), y Vos y León (2003), es un país que se ha caracterizado por cimentar sus exportaciones en un producto estrella, vinculándose con el mercado externo a través de un modelo primario exportador como ya han sido los casos del cacao, banano y actualmente el petróleo, y además por tener un crecimiento hacia afuera y dependiente de su sector externo lo que genera un grado de vulnerabilidad mayor.

En lo que corresponde al Coeficiente de Gini se puede observar que 
presenta una tendencia ascendente desde 1990 hasta aproximadamente el 2003. Como se mencionó en líneas anteriores, esta precarización en cuanto a desigualdad distributiva, corresponde en gran medida a la ejecución de políticas de corte neoliberal enfocadas hacia el control de la inflación, equilibrio en la balanza de pagos, y liberalización financiera, dejando al margen las cuestiones relacionadas con la distribución del ingreso. Es a partir del 2004, que se implementaron una serie de políticas sociales, tales como el aumento del gasto social en los sectores de salud, vivienda y educación que se logra revertir en alguna medida esta tendencia obteniéndose una reducción de la brecha de desigualdad de ingresos que se mantiene hasta el 2011.

\section{Pruebas de raíz unitaria y de estacionariedad}

Antes de proceder con la estimación del modelo, es preciso determinar el orden de integración de cada una de las series de tiempo con el objeto de establecer la especificación adecuada (nivel o primeras diferencias). Para ello se realizan las pruebas Dickey Fuller Aumentada (ADF) y Phillips-Perron (PP), las cuales permiten detectar la presencia de una raíz unitaria en las series de tiempo. La presencia de una raíz unitaria implica que un choque transitorio tendrá memoria infinita en la evolución de las variables. Así también, se aplica la prueba KwiatkowskiPhillips-Schmidt-Shin (KPSS) que detecta si una variable es o no estacionaria. Los resultados se presentan a continuación:

\section{Cuadro 1. Pruebas de raíz unitaria y de estacionariedad}

\begin{tabular}{|c|c|c|c|c|c|}
\hline \multirow{3}{*}{ Variable } & \multirow{3}{*}{$\begin{array}{c}\text { Especificación } \\
\text { de la Ecuación } \\
\text { de prueba }\end{array}$} & \multicolumn{3}{|c|}{ Estadística de Prueba } & \multirow{3}{*}{$\begin{array}{c}\text { Orden de } \\
\text { integración }\end{array}$} \\
\hline & & ADF & $\mathrm{PP}$ & KPSS & \\
\hline & & $\begin{array}{l}\text { Ho: Raíz } \\
\text { Unitaria }\end{array}$ & $\begin{array}{l}\text { Ho: Raíz } \\
\text { Unitaria }\end{array}$ & Ho: Estacionariedad & \\
\hline Gini & C & $-1,21$ & $-1,43$ & 0,19 & $\mathrm{I}(1) 0 \mathrm{I}(0)$ \\
\hline$\Delta$ Gini & C & $-2.72^{*}$ & $-2.62^{*}$ & 0,55 & $\mathrm{I}(0)$ \\
\hline PIBpc & C у TD & $-0,86$ & $-0,83$ & $0.15^{*}$ & $\mathrm{I}(1)$ \\
\hline$\triangle \mathrm{PIBpc}$ & C & $-3.50^{\star}$ & $-3.46^{*}$ & 0,34 & $\mathrm{I}(0)$ \\
\hline t_ratio & C y TD & $-2,93$ & $-2,93$ & 0,07 & $|(1) 0|(0)$ \\
\hline$\Delta$ t_ratio & C & $-5.88^{\star}$ & $-6.03^{*}$ & 0,19 & $\mathrm{I}(0)$ \\
\hline
\end{tabular}

Fuente: SWIID 3.0 y CEPAL.

Elaboración propia con estimaciones de Eviews.

* Rechazo de la hipótesis nula a un nivel de significancia de 5\%

$\Delta$ Operador de primeras diferencias. 


\section{Estimación del Modelo VAR}

Las pruebas de raíz unitaria y estacionariedad muestran que todas las variables tienen una raíz unitaria, son integradas de orden uno, por lo que se procede a diferenciarlas para volverlas estacionarias. De esta manera, se estima un modelo VAR estacionario de tres variables expresadas en primeras diferencias.

El horizonte del análisis corresponde desde 1993 hasta el 2011 para las tres variables, con esta información se procede a calcular el modelo VAR no restringido 3 .

$$
\begin{aligned}
& Y_{t}=C_{0}+\Gamma_{1} Y_{t-1}+\ldots . . \\
& +\Gamma_{\rho} Y_{t-\rho}+\Psi X_{t}+\eta_{t}
\end{aligned}
$$

donde:

$$
Y_{t}=\left[\begin{array}{c}
\text { PIBpc }_{t} \\
\text { Gini }_{t} \\
t_{-} \text {ratio }_{t}
\end{array}\right], \quad \eta_{t}=\left[\begin{array}{c}
\eta_{t}^{\text {PIBpc }} \\
\eta_{t}^{\text {Gini }} \\
\eta_{t}^{t_{t}^{\text {rataio }}}
\end{array}\right]
$$

3 Adicionalmente se incluyeron 2 rezagos, $\rho$, para cada variable.

\section{Resultados}

Como se ha señalado anteriormente, las principales herramientas de análisis del modelo VAR son las funciones de impulso respuesta y las descomposiciones de varianza. A continuación se muestran las estimaciones.

\section{Funciones de Impulso Respuesta}

Las Funciones de Impulso Respuesta (FIR) son una herramienta útil para evaluar la congruencia y la sensibilidad dinámica de las variables especificadas en el modelo; por tal razón, son muy eficientes para evaluar y proponer políticas económicas (Loría, 2007). Las FIR, muestran cómo los shocks en cualquiera de las variables afectan a las demás variables del modelo y con el tiempo retroalimentan a la misma variable original (Pindyck y Rubinfeld, 2001).

Las Funciones de Impulso Respuesta se estimaron para un horizonte de diez meses e incluyen intervalos de confianza de dos desviaciones estándar. Para que el análisis derivado de una FIR tenga validez estadística, su intervalo debe excluir al cero. A continuación se muestran las FIR del coeficiente de Gini ante cambios en el PIBpc y en la apertura comercial. 
Gráfico 2. Respuesta del Coeficiente de Gini frente a un choque en el PIBpc



Fuente: SWIID 3.0 y CEPAL.

Elaboración propia con estimaciones de Eviews.

Gráfico 3. Respuesta del Coeficiente de Gini frente a un choque en la t_ratio

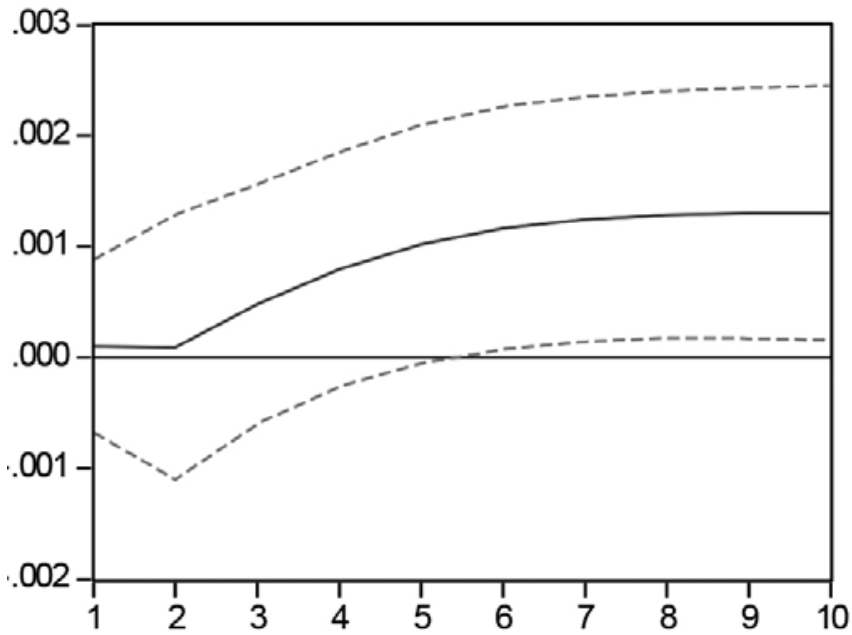

Fuente: SWIID 3.0 y CEPAL.

Elaboración propia con estimaciones de Eviews. 


\section{Descomposiciones de Varianza}

Los modelos VAR introducen las descomposiciones de varianza como instrumento para el análisis de las series de tiempo. Como se ha señalado, las FIR describen de qué manera (o con que signo) una variable se ve afectada a través del tiempo por choques no anticipados en otra variable, mientras que las descomposiciones de varianzas establecen en qué porcentaje una variable se ve afectada por choques en otra variable (Cuevas, 2008).

En el cuadro 2 se reportan las descomposiciones de varianza para el caso ecuatoriano para un horizonte estimado de 6 años. Centrando el análisis en la variable de desigualdad, se observa que el $21 \%$ de los cambios en la desigualdad se pueden explicar por la dinámica del sector externo de la economía. Así también, se visualiza evolución de la desigualdad distributiva tiene un marcado componente inercial.

\section{Cuadro 2. Descomposiciones de Varianza \% horizonte de tiempo 6 años}

\begin{tabular}{|c|c|c|c|}
\hline Variable Causal & PIBpc & Gini & $\begin{array}{c}\text { Apertura } \\
\text { Comercial }\end{array}$ \\
\hline Variable Dependiente & 98,33 & 0,65 & 1,02 \\
\hline Ginpc & 4,89 & 73,93 & 21,18 \\
\hline Apertura Comercial & 48,83 & 0,72 & 50,45 \\
\hline
\end{tabular}

Fuente: SWIID 3.0 y CEPAL

Elaboración propia con estimaciones de Eviews.

\section{Discusión y conclusiones}

Algunos autores avalan la tesis de que la liberalización y especialización del comercio no tendrían efectos negativos sobre la distribución del ingreso. Bajo esta premisa, los impulsores de este enfoque argumentan que el impacto distributivo de la liberalización externa probablemente sería neutral (o positivo, en el caso de zonas que tuvieran un excedente de mano de obra calificada), y que la desigualdad en la distribución del ingreso se mantendría estable en el largo plazo, dado que no existe una correlación fuerte entre la desigualdad y el crecimiento del producto (Deininger y Squire, 1997).

Tomemos en cuenta el gráfico 2 , en él se observa la función de impulso respuesta del Coeficiente de Gini frente a choques en el PIBpc. Para el caso ecuatoriano el ritmo de crecimiento de la economía no tendría 
efectos significativos sobre la distribución del ingreso, lo cual sugeriría que variaciones en el nivel del producto no aumentaría o reduciría la desigualdad distributiva en el Ecuador. Es decir, el crecimiento no garantizaría por sí mismo avances en cuanto a la reducción de la desigualdad.

Del gráfico 3 se desprende que una mayor apertura comercial, esto es mayor ritmo de crecimiento de las exportaciones e importaciones, aumentaría la brecha en los ingresos en la sociedad ecuatoriana. Esta evidencia se puede fundamentar en que el sector exportador en el Ecuador está integrado por un grupo reducido, el cual obtendría ingresos superiores fruto de esta actividad. Estos hallazgos son consistentes con los encontrados por Ponce y Vos, que señalan que durante la década de los noventa la desigualdad en la distribución del ingreso se ha incrementado por la liberalización del comercio, la cual ha consolidado el modelo primario exportador característico de la economía ecuatoriana (Ponce y Vos, 2012). Así también, una posible explicación se fundamenta en que la liberalización del comercio en los países con una desigual distribución del ingreso, tal es el caso de los países exportadores de productos primarios, ante un aumento en la exportación del producto estrella no le corresponde una mejora en términos distributivos del ingreso. Un trabajo reciente que recoge evidencia empírica para Argentina, Brasil, Chile, Colombia, Hong
Kong, India y México durante el período de 1980-1990, identifica que a un aumento en la integración económica le corresponde en paralelo un incremento en la desigualdad de ingresos (Goldberg y Pavnick, 2007). Así, esta evidencia confirma que una mayor apertura comercial no favorece al estrato de la población con menores niveles de ingreso. Como indican Székely y Sámano "podemos caracterizar los años 1980-2000 en América Latina como un período donde la apertura comercial se asocia con efectos de desigualdad creciente, pero estos no fueron lo suficientemente fuertes de tal manera que no afectan a la estructura de la economía" (Székely y Sámano, 2011).

Behrman, Birdsall y Székely (2001), realizan una investigación sobre el efecto de la liberalización comercial y financiera sobre la desigualdad y pobreza en América Latina entre 1977 y 2000. Utilizando datos sobre las encuestas de hogares de 17 países, y estimando un modelo de datos de panel con efectos fijos se encuentran dos hallazgos interesantes: Primero, la liberalización financiera tiene un impacto positivo y estadísticamente significativo sobre la desigualdad, esto es, empeora la distribución del ingreso. Explican los autores que la liberalización financiera permite un flujo mayor de capitales hacia la región, lo cual tiende a reducir el precio del capital; tomando en cuenta que la relación entre el capital y la mano de obra calificada 
es complementaria, y con la mano de obra no calificada es sustitutiva, se espera que la demanda trabajadores educados aumente en oposición a la demanda de trabajadores no educados. De este modo, la liberalización financiera ocasiona que la brecha salarial entre los trabajadores aumente, repercutiendo en un empeoramiento de la distribución del ingreso. Segundo, la liberalización comercial parece no haber tenido efectos negativos sobre la distribución del ingreso; sin embargo, los autores señalan que el coeficiente asociado a la apertura comercial es no significativo.

Del cuadro 2 se aprecia que los choques originados por la apertura comercial seis años después explican el $21.28 \%$ de las variaciones en el coeficiente de la distribución del ingreso. Esta interpretación es consistente con los resultados proporcionados por la FIR, representada en el gráfico 3. Además, la dinámica presentada por el Coeficiente de Gini tiene un marcado componente inercial, ya que el comportamiento pasado de la distribución del ingreso explica el $73.93 \%$ del comportamiento futuro de esta variable.

En el trabajo de Cornia, la evidencia empírica sugiere que una mayor apertura externa conduce a un incremento en la desigualdad de ingresos (Cornia, 2011). El autor argumenta que el empeoramiento de la desigualdad entre 1980-2000 se debió al bajo crecimiento del PIB que caracterizó a la "década perdida" en algunas regiones, tal fue el caso de América Latina. Hallazgos similares son los encontrados por Székely y Sámano (2012), quienes realizan una estimación entre la apertura comercial y la distribución del ingreso para un período de 30 años (1980-2010) para 18 países de América Latina ${ }^{4}$, del cual se desprende que "una mayor apertura comercial se asocia a incrementos contemporáneos en la desigualdad en la región, y que la apertura comercial contribuyó, junto con otros factores, al aumento de la desigualdad durante las décadas de los ochenta y noventa, pero una vez que fue implementada totalmente, no llevó a mayores incrementos en la desigualdad que se atribuyen a otros factores tales como la diferencia en los niveles educativos entre la población".

Así mismo Vos, Ganuza, Robinson y Morley, estudian el comportamiento de 16 países latinoamericanos entre 1980-2005 y la relación entre la liberalización comercial, el crecimiento económico y la desigualdad. Para ello formulan un modelo

4 La investigación incluye estadísticas de: Argentina, Bolivia, Brasil, Chile, Colombia, Costa Rica, Ecuador, El Salvador, Guatemala, Honduras, México, Nicaragua, Panamá, Paraguay, Perú, República Dominicana, Uruguay y Venezuela. Utilizan el Índice de Gini como medida de la desigualdad en la distribución del ingreso y el valor medio de los aranceles para representar el grado de apertura comercial. 
de equilibrio general computarizado (CEG)5. Del modelo estimado exponen que como consecuencia del proceso de apertura económica al mercado mundial, el crecimiento del producto ha estado liderado por las exportaciones en todos los países de la región, sin embargo, el crecimiento económico no aumentó significativamente tras la apertura comercial. En cuanto a la desigualdad y pobreza, los autores señalan que han habidos avances en estas áreas, y que la liberalización comercial condujo a un incremento en los salarios y una mejora en términos de lucha contra la pobreza (Vos et al., 2004).

Este trabajo ofrece una perspectiva a corto plazo para el análisis de la relación entre la distribución del ingreso y la apertura comercial en el Ecuador durante las últimas dos décadas. El análisis de las series temporales a partir del modelo de vectores autorregresivos utilizado en este estudio arroja tres conclusiones importantes. La primera, la apertura comercial determina la evolución de la desigualdad del ingreso. A un aumento en el flujo de comercio externo corresponde un aumento en la desigualdad distributiva. Segundo, el crecimiento del producto de la economía y el coeficiente de apertura comercial presentan una relación directa positiva. Esto es, una mayor

5 Un modelo CEG se define como un conjunto de ecuaciones simultáneas, muchas de las cuales son no lineales y no existe una función objetivo. Las ecuaciones definen el comportamiento de los diferentes actores.

Retos V (I0): 2015

(C) 2015, Universidad Politécnica Salesiana del Ecuador apertura comercial conlleva a un incremento en el PIB por habitante. Por último, la evidencia empírica si bien logra identificar algunas relaciones entre las variables, estas no son lo suficientemente robustas, por tal motivo se sugiere avanzar en esta línea de tal forma que se fortalezcan estas relaciones de causalidad.

\section{Bibliografía}

Acosta, A. (2005). Breve Historia Económica del Ecuador. Ecuador: Corporación Editora Nacional.

Behrman, J; Birdsall, N. \& Székely, M. (2001). Pobreza, Desigualdad, y Liberalización financiera en América Latina. Working Paper, Banco Interamericano de Desarrollo, Research Department, núm. 449.

Cuevas, V. (2008). Inflation, Output and Economic Policy in Mexico. Investigación Económica, 67(265), 85 120, julio-septiembre.

Cornia, G. (2011). Economic integration, inequality and growth: Latin America vs. the European economies in transition. Review of Economics and Institutions, 2(2). Italia: Universidad de Perugia.

Deininger, K. \& Squire, L. (1997). Economic growth and income inequality: reexamining the links. Finance and Development, 34, Estados Unidos: FMI.

Goldberg, K. \& Pavcnik, N. (2007). Distributional effects of globalization in developing countries. Journal of Economic Literature, vol. 45, núm. 1, Estados Unidos: American Economic Association. 
Gujarati, D. N. \& Porter, D. C. (2010). Econometría. México: McGrawHill.

Loría, E. (2007). Econometría con Aplicaciones. México: Pearson Prentice Hall.

Maddala, G. (1996). Introducción a la Econometría. México: Prentice Hall Hispanoamericana.

Morán, D. (2014). Determinantes de la Inflación en Ecuador. Un análisis econométrico utilizando modelos VAR. Economía y Sociedad, 18(31). México: Universidad Michoacana de San Nicolás de Hidalgo.

Ordóñez, D. (2006). Ecuador: comercio exterior y coeficiente de apertura de la economía 2000-2004. Observatorio de la Economía Latinoamericana, 61. http://www.eumed.net/cursecon/ecolat/index.htm (14 de marzo de 2013).

Pindyck, R. S. \& Rubinfeld, D. L. (2001). Econometría: Modelos y Pronósticos.
México: McGraw-Hill.

Ponce, J., \& Vos, R. (2012). Redistribution without Structural Change in Ecuador: Rising and Falling Income Inequality in the 1990s and 2000s. Unu Wider, Working Paper No. 2012/12.

Sims, C. (1980). Macroeconomics and Reality. Econometrica, 48(1). Estados Unidos: The Econometric Society.

Székely, M. \& Sámano, C. (2012). Did Trade Openness Affect Income Distribution in Latin America? Unu Wider, Working Paper No. 2012/03.

Vos, R; Ganuza, E; Robinson, S. \& Morley, S. (2004). ¿Quién se beneficia del Libre Comercio? Promoción de exportaciones en América Latina y el Caribe en los 90. Colombia: Alfaomega.

Vos, R. \& León, M. (2003). Dolarización, dinámica de exportaciones y equidad: ¿cómo compatibilizarlas en el caso de Ecuador? Quito: Unidad de Información y Análisis del SIISE.

Recepción: 14-08-2015 - Aceptación: 27-11-2015. 